\title{
Dopplershifts in the solar transition region
}

\author{
C. Gontikakis ${ }^{1}$, H. C. Dara ${ }^{1}$, C. E. Alissandrakis ${ }^{2}$, Th. G. Zachariadis ${ }^{1}$, and J.-C. Vial ${ }^{3}$ \\ 1 Research Center for Astronomy and Applied Mathematics, Academy of Athens, 14 Anagnostopoulou St., \\ 10673 Athens, Greece \\ 2 Section of Astro-Geophysics, Department of Physics, University of Ioannina, 45110 Ioannina, Greece \\ 3 Institut d'Astrophysique Spatiale, Unité Mixte CNRS-Université Paris XI, Bât. 121, 91405 Orsay, France
}

Received 13 April 2000 / Accepted 28 August 2001

\begin{abstract}
We study the dynamics of the quiet sun transition region, using observations obtained with the SOHO CDS/NIS and SUMER spectrographs. We examine the morphology of the network as a function of temperature and we compare the intensity features with those of the dopplergrams. The velocity distributions have a different behaviour for the bright features which outline the network and the dark ones, located in the internetwork. A redshift and a smaller standard deviation are observed for the bright feature distributions relative to the dark ones. It should be mentioned that the internetwork is also statistically redshifted, with the exception of the He I line. Velocity distributions from different lines are compared.
\end{abstract}

Key words. Sun: transition region - line formation

\section{Introduction}

The EUV lines emitted by the solar transition region plasma show a systematic redshift, which was first observed with Skylab and the Orbiting Solar Observatory (OSO 8) (Doschek et al. 1976; Bruner et al. 1976 respectively). During the last decades, this observation has been confirmed by space missions and rocket launches: OSO 8 (Roussel-Dupré \& Shine 1982), the High Resolution Telescope and Spectrograph (HRTS, Athay \& Dere 1989; Brekke 1993; Achour et al. 1995), the Laboratory for Atmospheric and Space Physics (LASP) EUV Coronal Spectrometer (Hassler et al. 1991) and, lately, the Solar and Heliospheric Observatory (SOHO, Domingo et al. 1995). Redshifts were also observed with the International Ultraviolet Explorer (Ayres et al. 1983) and, recently, from the Hubble Space Telescope (Wood et al. 1996, 1997) in the spectra of late-type stars, showing that this is not characteristic of our star only. The measurements of absolute velocities as a function of the formation temperature of the lines are difficult, since, on-board most instruments there is no zero velocity reference. Chromospheric lines have been used as a reference (Doschek et al. 1976; Athay \& Dere 1989; Brekke 1993; Chae et al. 1998; Teriaca et al. 1999), for the measurement of transition region lines on the disk. The redshift of these chromospheric lines is considered to be negligible as in the case of the photosphere (Samain 1991). Limb motions are also considered negligible, because, statistically, the flow is perpendicular

Send offprint requests to: C. Gontikakis,

e-mail: cgontik@cc.uoa.gr to the solar surface and the horizontal motions are canceled (Doschek et al. 1976; Hassler et al. 1991, 1999; Peter \& Judge 1999). The use of an on-board calibration (Hassler et al. 1991) probably provides the most accurate results. A general conclusion of the observers, up to now, is that the redshift increases with temperature from $10^{4} \mathrm{~K}$ to $10^{5} \mathrm{~K}$, reaches a maximum value of $\simeq 10 \mathrm{~km} \mathrm{~s}^{-1}$ at $10^{5.3} \mathrm{~K}$ (Teriaca et al. 1999; Peter \& Judge 1999) and then decreases. However, the dynamic behaviour of the upper transition region, with plasma temperatures higher than $\simeq 200000 \mathrm{~K}$, is not clear. Recent studies, in contrast with older ones (Brekke 1993; Achour et al. 1995; Chae et al. 1998), using few (Hassler et al. 1999; Dammasch et al. 1999) as well as many lines (Peter 1999; Peter \& Judge 1999; Teriaca et al. 1999), infer that for temperatures higher than $10^{5.7} \mathrm{~K}$ the spectrum is blueshifted.

However, the transition region is far from being uniform and steady, while the relation between redshifts and formation temperatures is statistical. Variations may occur within a few seconds (Porter et al. 1984; Cheng 1991) and in a spatial length of a few arcseconds (Judge et al. 1997). Oscillations in the range of $3-10 \mathrm{mHz}$ have also been reported in the upper chromosphere and low transition region (e.g. Judge et al. 1997; Gouttebroze et al. 1999; Wikstøl et al. 2000).

In this article we study the morphology and the smallscale motions in the transition region in a quiet sun region. In Sect. 2 we describe our observations and the data treatment. Section 3 presents our results which we discuss in Sect. 4. 


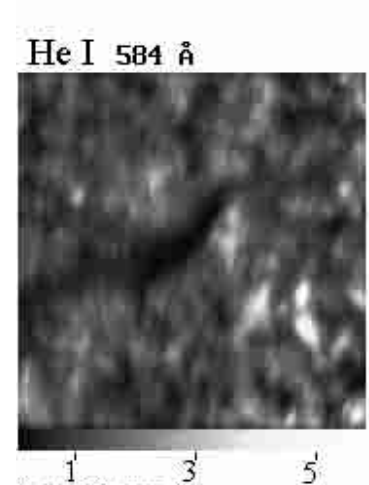

\section{O IV 554 ค̊}

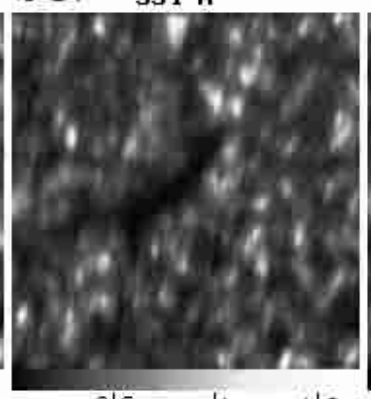

Ju1 $281996 \quad 16: 38: 41$
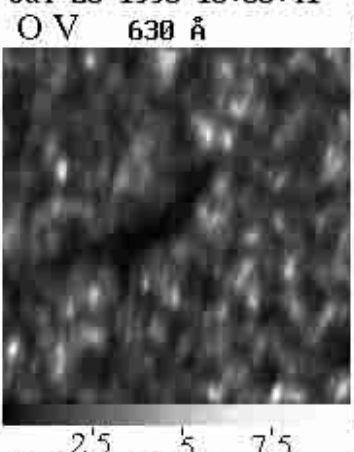

$\mathrm{NeVI} 563 \stackrel{\circ}{\circ}$

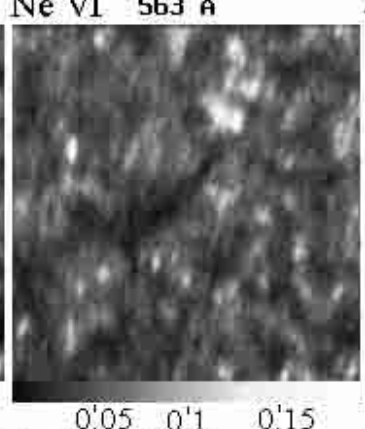

$\operatorname{Mg}$ IX 368 ค̊

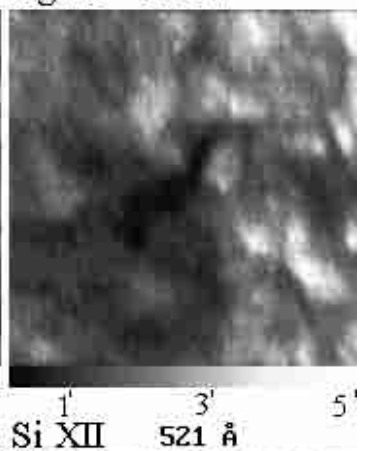

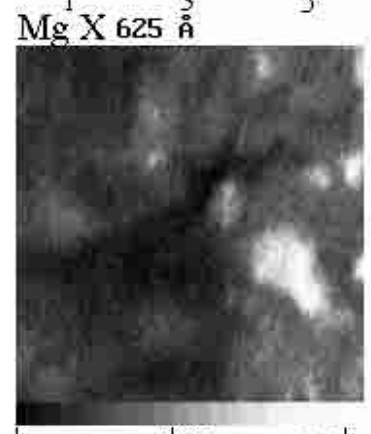

0.02

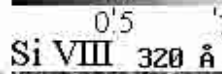

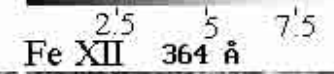

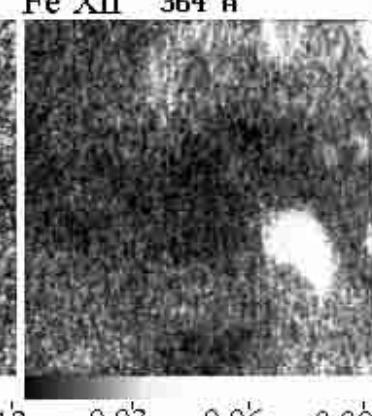

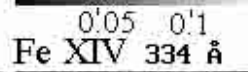

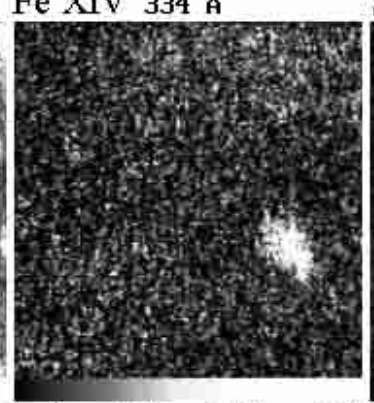

$0.03 \quad 0.05 \quad 0.07$

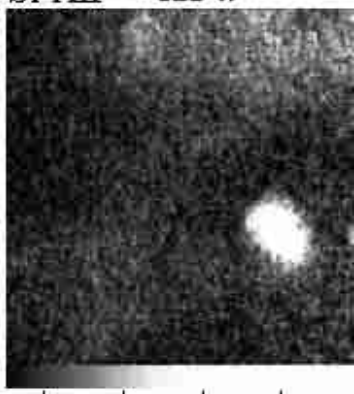

$0.03 \quad 0.05 \quad 0.07$

Fig. 1. Intensity images, in the He I $584.33 \AA$, O IV $554.52 \AA$, O v $629.73 \AA$, Ne vi $562.83 \AA, \mathrm{Mg}$ IX $368.06 \AA, \mathrm{Mg}$ x $624.94 \AA$, Si VIII $319.83 \AA$, Fe XII $364.47 \AA$, Fe XIV $334.17 \AA$, Si XII $520.67 \AA$ lines, observed on July 28, 1996. The field of view is $240^{\prime \prime} \times 240^{\prime \prime}$. Peak intensity units are $10^{3} \mathrm{erg} \mathrm{s}^{-1} \mathrm{~cm}^{-2} \mathrm{sr}^{-1} \AA^{-1}$. The images are presented in order of increasing temperature of ion formation from left to right, continuing in the second row. The network pattern, in lines hotter than Ne VI, cannot be detected. In the lower right part of the Mg x, Fe XII, Fe XIV and Si XII line images one can see a coronal loop.

\section{Observations}

The observations were obtained during a SOHO/VLA filament campaign in July 1996 (Alissandrakis et al. 1997; Chiuderi-Drago et al. 1998). In this work we used observations of the 28th of July, from the Coronal Diagnostic Spectrometer (CDS, Harrison et al. 1995) and the Solar Ultraviolet Measurements of Emitted Radiation (SUMER, Wilhelm et al. 1995) on SOHO. The instruments were pointed on a quiet region around a filament, located at $41^{\circ} \mathrm{W}$ and $41^{\circ} \mathrm{N}$. The region was at a heliocentric distance of $\cos \theta=0.6$. CDS provided, with its Normal Incidence Spectrometer (NIS), a sequence of four observations over approximately four hours. The lines were selected from the two wavelength bands of the instrument (308-381 $\AA$ and 513-633 $\AA$ ).

The data were obtained by scanning the field of view with the NIS slit in a direction perpendicular to its length, a procedure which provides "raster" images. In Table 1 we give all the information about the rasters. The first and last rasters of the second day (s3842 and s3845) give more spatial information, since they cover a larger field of view, while the intermediate ones (s3843 and s3844) provide more spectral windows, that is more lines. Figure 1 shows intensity images in the different lines with the large field of view.

Moreover, we analysed SUMER observations of July 28. They are in the form of intensity images and dopplergrams computed from the first moment of the line profile. These data are compressed on-board using the routine "Gauss fit B 2 moment" (Dammasch 1992; Wilhelm et al. 1995). They include four rasters of the same regions as the one of CDS. SUMER scans the region from east to west and then from west to east for every set of spectral lines, with $8 \mathrm{~s}$ exposure time, using the $\mathrm{KBr}$ part of detector A. The images are larger in the North South direction since the slit is $1^{\prime \prime} \times 300^{\prime \prime}$, whereas the length of the CDS slits we used was $240^{\prime \prime}$. In Table 2 we give more information about the SUMER observations.

\section{Data reduction}

\subsection{CDS data}

The NIS data were calibrated using the CDS software in order to obtain absolute intensity values (in

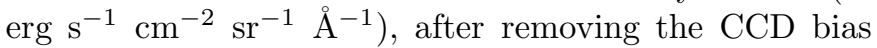
and the cosmic-ray traces. Two of the systematic errors which were corrected in the dopplergrams are:

a. the tilt of the spectral lines on the detector, which produces a north-south gradient on the dopplergrams, and b. the shift of the wavelength zeropoint with the scan mirror position, which is responsible for an east-west gradient on the dopplergrams (Thompson \& Brekke 2000). Furthermore, we excluded two pixel rows from the top 
Table 1. The CDS/NIS observations.

\begin{tabular}{llccccc}
\hline $\begin{array}{l}\text { Raster } \\
\text { name }\end{array}$ & Date & Start time & End time & $\begin{array}{c}\text { Exposure } \\
\text { time }(\mathrm{s})\end{array}$ & Slit & $\begin{array}{c}\text { field of } \\
\text { view }\end{array}$ \\
\hline s3842 & July 28 & $15: 46: 19$ UT & $17: 31: 03$ UT & 45 & $2^{\prime \prime} \times 240^{\prime \prime}$ & $240^{\prime \prime} \times 240^{\prime \prime}$ \\
s3843 & July 28 & $17: 31: 33$ UT & $18: 17: 52$ UT & 30 & $4^{\prime \prime} \times 240^{\prime \prime}$ & $122^{\prime \prime} \times 240^{\prime \prime}$ \\
s3844 & July 28 & $18: 19: 08$ UT & $19: 05: 58$ UT & 30 & $4^{\prime \prime} \times 240^{\prime \prime}$ & $122^{\prime \prime} \times 240^{\prime \prime}$ \\
s3845 & July 28 & $19: 07: 25$ UT & $20: 52: 12$ UT & 45 & $2^{\prime \prime} \times 240^{\prime \prime}$ & $240^{\prime \prime} \times 240^{\prime \prime}$ \\
\hline
\end{tabular}

Table 2. SUMER observations.

\begin{tabular}{|c|c|c|c|c|}
\hline Raster & Start time & End time & $\begin{array}{l}\text { field of } \\
\text { view }\end{array}$ & Spectral lines $(\AA)$ \\
\hline First & 15:54:24 UT & 16:08:08 UT & $100^{\prime \prime} \times 300^{\prime \prime}$ & $\begin{array}{l}\text { Ne VIII } 770,780 \\
\text { C IV } 1548,1550\end{array}$ \\
\hline Second & 16:08:08 UT & 16:22:18 UT & $100^{\prime \prime} \times 300^{\prime \prime}$ & $\begin{array}{l}\text { Ne VIII } 770,780 \\
\text { C IV } 1548,1550\end{array}$ \\
\hline Third & 16:22:18 UT & 16:36:03 UT & $100^{\prime \prime} \times 300^{\prime \prime}$ & $\begin{array}{l}\text { Si IV } 1394,1403 \\
\text { O IV } 1401,1405\end{array}$ \\
\hline Fourth & 16:36:03 UT & 16:49:52 UT & $100^{\prime \prime} \times 300^{\prime \prime}$ & $\begin{array}{l}\text { Si IV } 1394,1403 \\
\text { O IV } 1401,1405\end{array}$ \\
\hline
\end{tabular}

and the bottom of each image to avoid effects due to the edge of the slit.

From the CDS data, we created intensity images and dopplergrams. For the study of the morphology (Sect. 4.1), we calculated the total intensity of each pixel in order to create total intensity images for each line. This method makes easier the treatment of a blend of four lines from the same element (O IV) at $554 \AA$, which is difficult to face with a Gaussian fitting routine.

For the study of the motions of the network elements (Sect. 4.2), we selected the brightest, Gaussian shaped lines among those which are formed in the transition region (He I $584.33 \AA$, O v $629.73 \AA$, Ne vi $562.83 \AA$, O III $599.59 \AA$ ). For these lines we computed dopplergrams using a Gaussian fitting routine for each profile of the image. The average velocity of each dopplergram is set equal to zero. We also computed them with two complementary methods: the first moment of the profiles and the position of the peak intensity of each profile. The three methods give similar results, even if the two latter ones are less accurate than the gaussian fit method. In the case of blended lines (in our case Ne VI $562.83 \AA$ ) the results of the method of the peak intensity position are in agreement with those of the Gaussian fit.

Moreover, CDS data need a careful treatment of the statistical errors in order to deduce reliable dopplershifts. The weight function adopted for the Gaussian fitting routine for each spectral point of the profiles is the inverse of the square of the instrumental $\sigma=\sqrt{2|N|+R^{2}}$ (CDS software note No. 49, W. Thompson 1998), where $N$ is the number of photon-events per spectral pixel and $R$ is the readout noise ( 1 photon-event/pixel). The statistical noise is due to the Poisson distribution of the photon interactions with the NIS detector known as Viewfinder Detector Subsystems (VDS) and to the fluctuation in the amount of amplification in the detector system, known as the Pulse Height Distribution (PHD). The latter effect is assumed to be comparable to the Poisson noise, hence the factor of two in the expression of $\sigma$. However, the variability of the data is less than the value of the instrumental $\sigma$. This is due to the fact that a photon-event is smeared over more than one pixel, so that the noise count for this pixel is the contribution of the counts from more than one. This effect occurs along the slit and along the dispersion axis and may affect the results of the Gaussian fitting of the profiles.

We made tests by adding noise to the data in order to compensate the above mentioned smoothing effect and we concluded that the results of the Gaussian fits are reliable. Therefore, we can determine the error of the dopplershifts by using the $\sigma_{\mathrm{VEL}}$, computed from the Gaussian fitting routine. The uncertainty of the dopplershifts for each spectral line is shown in Table 3 . For the spectral lines of Ne VI $562.83 \AA$, O III 599.59 $\AA$ and $\mathrm{Mg}$ IX $368.06 \AA$ we took into account only the profiles where the peak intensity is larger than 0.25 photon events pixel ${ }^{-1} \mathrm{~s}^{-1}$ (Thompson \& Brekke 2000). Below this value, the profiles are dominated by noise and the uncertainties in the velocities are very large.

Statistical analysis of a large set of quiet sun CDS/NIS data showed that the dopplergrams of some lines $(\mathrm{O} \mathrm{V}$ $629.73 \AA$, He I $584.33 \AA, \mathrm{Mg}$ IX $638.06 \AA$ ) are correlated with the corresponding intensity morphology in a way that indicates an instrumental error, rather than a solar phenomenon (Haugan 1999). The gradient of the intensity along the slit introduces a variation in the dopplershift which, however, is difficult to detect on the dopplergrams. Thus, it seems that bright features correspond to blueshifts at their northern edge and redshifts at their southern edge. This deformation can be partially removed 
Table 3. Spectral lines used for the present study. The left part shows the CDS/NIS lines and the right one the SUMER lines. In addition to the wavelength and the temperature of formation, the table gives the $1 \sigma$ error for the velocity in the lines chosen for Doppler analysis. The SUMER line O IV $1405 \AA$ is blended with a S IV line. The ions marked with a star (*) are the ones used for the morphology study. Ionization equilibrium temperatures are from Arnaud \& Rothenflug (1985) except for the iron lines which are from Arnaud \& Raymond (1992).

\begin{tabular}{llll|llll}
\hline \hline Ion & $\begin{array}{l}\lambda \\
{[\AA]}\end{array}$ & $\begin{array}{l}\log T \\
{[\mathrm{~K}]}\end{array}$ & $\begin{array}{l}\text { error bar } \\
{\left[\mathrm{km} \mathrm{s}^{-1}\right]}\end{array}$ & Ion & $\begin{array}{l}\lambda \\
{[\AA]}\end{array}$ & $\begin{array}{l}\log T \\
{[\mathrm{~K}]}\end{array}$ & $\begin{array}{l}\text { error bar } \\
{\left[\mathrm{km} \mathrm{s}^{-1}\right]}\end{array}$ \\
\hline He I$^{*}$ & 584.33 & 4.3 & 6 & Si IV & 1393.76 & 4.8 & 4 \\
O III & 599.59 & 5.0 & 18 & Si IV & 1402.77 & 4.8 & 4.3 \\
O IV $^{*}$ & 554.52 & 5.2 & $\ldots$ & C IV & 1548.202 & 5.0 & 6.6 \\
O v $^{*}$ & 629.73 & 5.4 & 7 & C IV & 1550.774 & 5.0 & 8 \\
Ne VI $^{*}$ & 562.83 & 5.6 & 27 & O IV & 1401.156 & 5.3 & 6 \\
Mg IX $^{*}$ & 368.06 & 5.9 & 27 & O IV & 1404.810 & 5.3 & $\ldots$ \\
Mg X & 624.94 & 6.0 & $\ldots$ & Ne VIII & 770.428 & 5.8 & 5 \\
Si VIII* & 319.826 & 5.9 & $\ldots$ & Ne VIII & 780.324 & 5.8 & 7.2 \\
Fe XII & 364.467 & 6.1 & $\ldots$ & & & & \\
Fe XIV* & 334.172 & 6.3 & $\ldots$ & & & & \\
Si XII & 520.492 & 6.3 & $\ldots$ & & & & \\
\hline \hline
\end{tabular}

if we correct each pixel on the dopplergram by a quantity proportional to the gradient of the intensity along the slit. The resulting dopplergrams are smoother, but their morphology is not modified.

\subsection{SUMER data}

The calibration of the SUMER data has been carried out with the appropriate software and transforms the counts per sec into intensity units $\left(\mathrm{erg} \mathrm{s}^{-1} \mathrm{~cm}^{-2} \mathrm{sr}^{-1} \AA^{-1}\right)$ so that we can compare them with the CDS data. The "Gauss fit B 2 moment", applied on-board to the SUMER data subtracts the background before fitting each profile to a Gaussian.

The SUMER dopplergrams were corrected for geometric distortions. As our data were compressed on-board, we couldn't use directly the SUMER software (Moran routine) and we used correction parameters provided by W. Curdt (private communication). For the wavelength calibration we used the dispersion of the spectrograph to get the values in $\mathrm{km} \mathrm{s}^{-1}$, and we considered the average velocity of each image equal to zero. Therefore, in this work, we deal with relative velocities. Moreover, in order to have results comparable with the ones of CDS, the SUMER pixel size was binned to the CDS pixel size.

In order to estimate the uncertainties in the SUMER dopplergrams calculated on-board we used the following procedure:

For each spectral line, we applied a Monte-Carlo simulation to create a number of gaussian profiles, with zero dopplershifts and a total number of counts corresponding to the total counts of the dark regions. Then we computed the dopplershift of each profile by the method of the first moment. The calculated RMS of these dopplershifts, given in Table 3, represents the uncertainties. These are maximum values since the dark, low count regions were used.

\subsection{Selection of network regions (masks)}

To compute the average dopplershift of the network structures from the CDS data, we created image masks based on the $\mathrm{O} \mathrm{V}$ images of integrated intensity. We selected $30 \%$ of the pixels of highest intensity and $25 \%$ of the ones of lowest intensity. Figure 2 shows the masks for the data which we call high intensity mask and low intensity mask respectively. We applied these masks also to the low transition region lines of He I and Ne VI, where the network morphology is similar. Applying these intensity masks on the dopplergrams, we separated the profiles into two categories, network and internetwork ones. In Fig. 3 we show the histogram of the intensity of the $\mathrm{O} v$ image and the selection done by the masks. Let us note that before the above procedure, we removed part of the image corresponding to the filament and the coronal loop seen in Fig. 2, so that they don't influence our results on network dynamics. These masks are also applied to the He I image where the network has a similar morphology.

The dynamics of the network pattern was also studied with the SUMER data. We created again high and low intensity masks from the image of Si IV $1394 \AA$. We selected this line from the SUMER data because the network is clearly visible. Again, we selected $30 \%$ of the brightest pixels to form the high intensity mask and $25 \%$ of the darker pixels for the low intensity mask. In Fig. 2 we show the Si IV masks and in Fig. 3 the histogram of the Si IV $1394 \AA$ peak intensities. The filament is invisible in the Si IV lines because there is no absorption by the neutral hydrogen in lines above the Lyman continuum (Chiuderi-Drago et al. 1997), so we consider that the motions inside the filament do not influence the Si IV lines and this is why we did not mask the pixels corresponding to the filament area. However, in the case of Ne VIII where the filament is visible, we mask it before the use of the high and low intensity masks. The Si IV $1394 \AA$ masks were also applied to the 


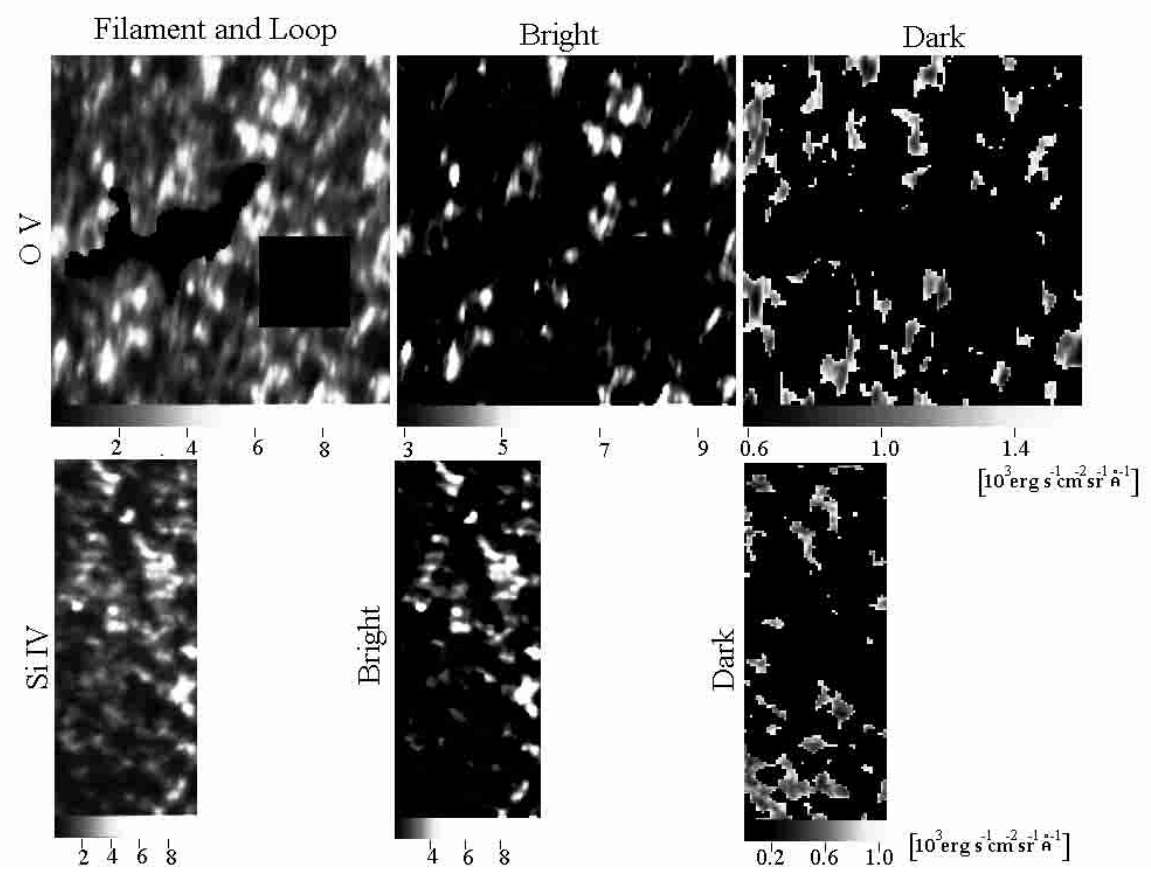

Fig. 2. Images of O v (first row) and Si IV (second row). The first panel shows the O v $630 \AA$ image with the filament and loop regions masked. The second and third columns correspond to the masks of high and low intensity regions, depicting the bright and dark points of the network. Peak intensity units are in $\operatorname{erg~s}^{-1} \mathrm{~cm}^{-2} \mathrm{sr}^{-1} \AA^{-1}$.
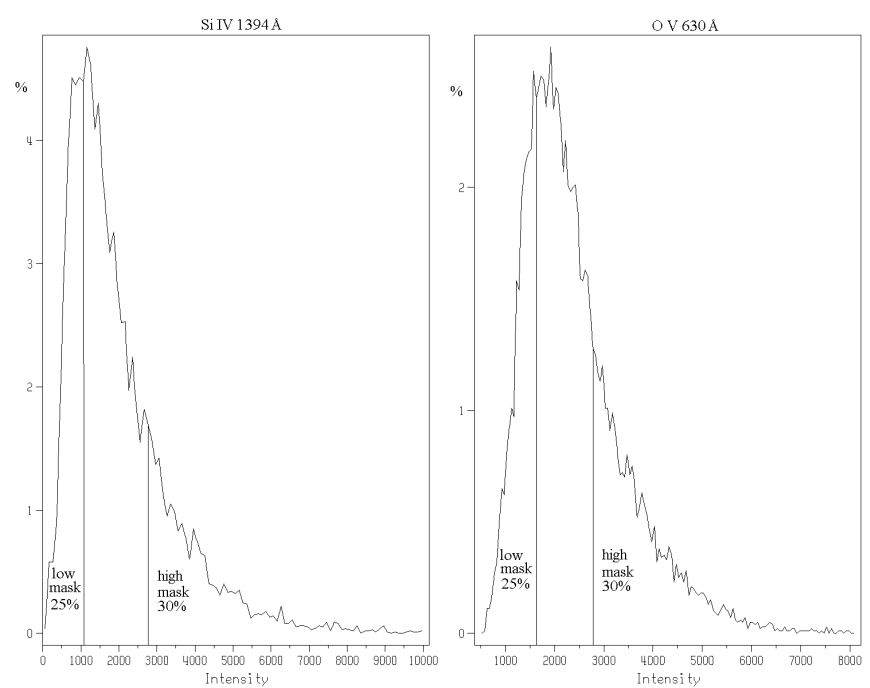

Fig. 3. Histograms of the peak intensities of the Si IV $1394 \AA$, O v $630 \AA$ lines showing the intensity ranges associated with the masks. Units are in $\operatorname{erg~s}{ }^{-1} \mathrm{~cm}^{-2} \mathrm{sr}^{-1} \AA^{-1}$.

SUMER lines of the ions C IV and O IV because they have a similar morphology.

\section{Results}

\subsection{Morphology}

The change of the network structure with temperature was studied using CDS data (Table 3). The sequence of CDS images from raster s3842 (Fig. 1) shows clearly the bright network features and the filament in the low transition

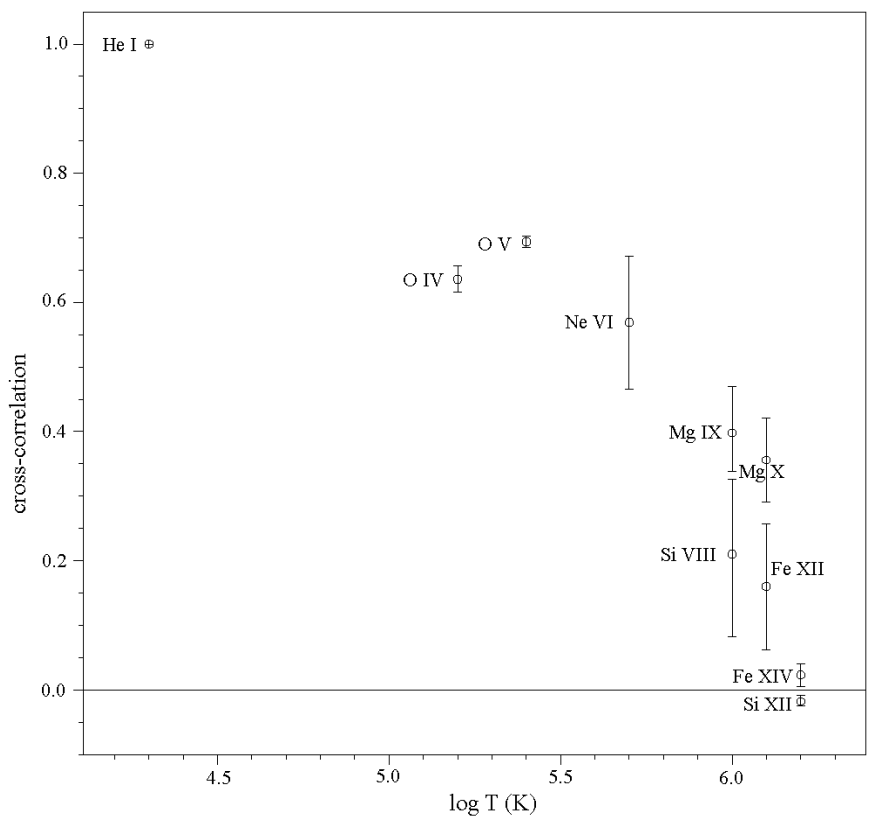

Fig. 4. Cross-correlation between the images of the s3842 raster and the He I image of the same raster. The correlation is good up to the Ne VI line $(60 \%)$ and decreases abruptly in the coronal lines ( $\mathrm{Mg} \mathrm{IX}, \mathrm{Mg} \mathrm{X}, \mathrm{Fe}$ XII etc.). The filament and the coronal loop have been masked out.

region lines (He I, O IV, O v, Ne VI). In Mg IX, the network is hardly visible, in the lines with higher formation temperatures (Mg X, Si XII etc.) the network and the filament are not detectable, while a coronal loop becomes prominent. The gradual disappearance of the network pattern 

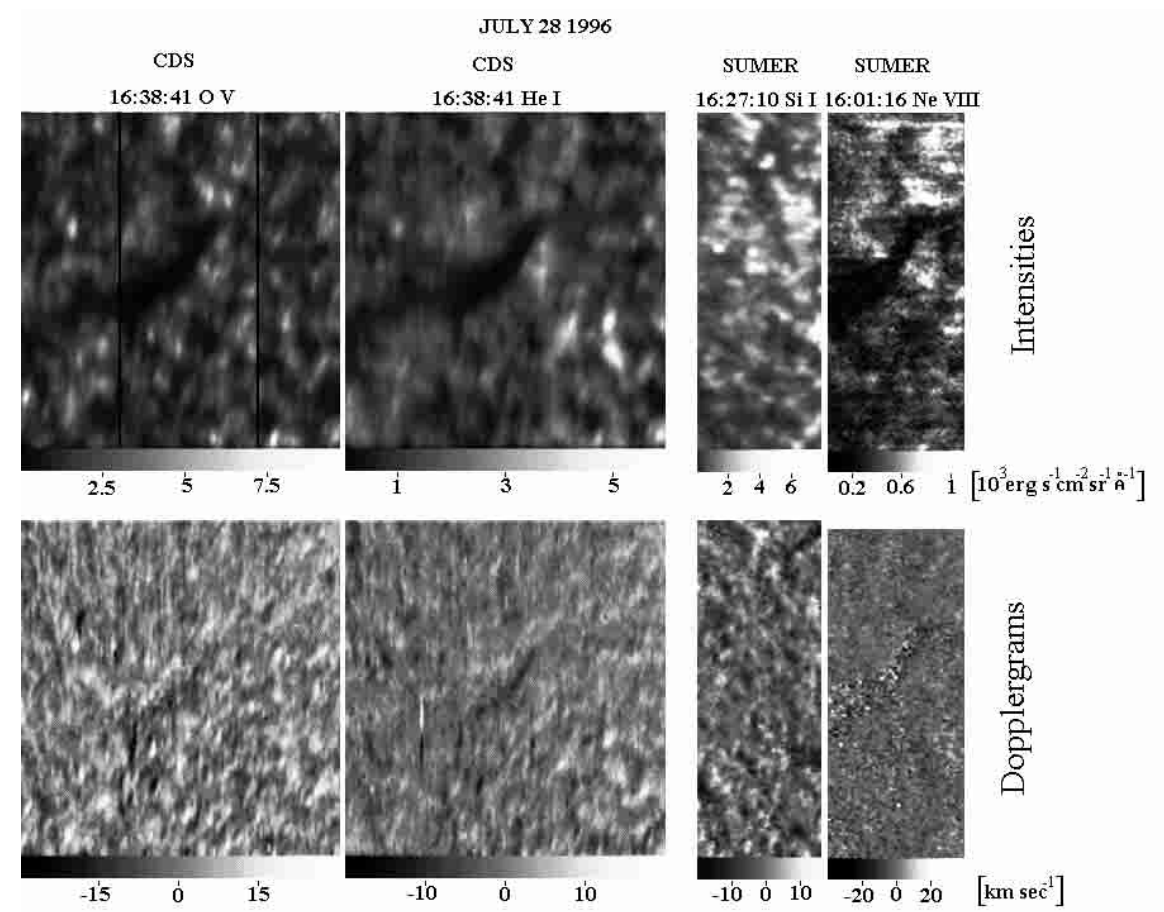

Fig. 5. Intensity images (first row) and dopplergrams (second row) for O v and He I lines from CDS and Si IV and Ne VIII from SUMER. The CDS images were computed from Gaussian fits of the profile of each pixel. The vertical black lines in the $\mathrm{O} v$ image delimit the SUMER field of view. Positive values correspond to redshisfts. Smoothing along the slit (see 3.1) may be the reason for the elongation of the features in the CDS images.

from the chromosphere to the corona has already been pointed out on the basis of Skylab observations (Reeves 1976) and more recently with SOHO (Patsourakos et al. 1999). In order to give a quantitative picture, we computed the cross-correlation coefficient, that is the covariance of the intensity of each image and the intensity of the He I one, normalized by the product of the intensity standard deviation of the two images (Fig. 4). To avoid the influence of the filament and the coronal loop, the corresponding pixels were masked out (upper left Fig. 2). As expected, the cross-correlation coefficient decreases as we move to the corona and drops below 0.5 at $\log T=6$. In the higher temperature lines (Fe XII, Fe XIV, Si XII) the cross-correlation coefficient is near zero. In these lines, the image noise is considerable and may influence the value of the cross-correlation coefficient; therefore we should estimate its influence.

The fractional noise is $f_{N}=\frac{\sqrt{2|N|+R^{2}}}{N}$, where $N$ is the sum of photon-events along the profile of each pixel. We computed the average noise for each of the images of Fig. 1. Then, we introduced in the He I image different noise levels (corresponding to the computed ones), using artificial Poisson noise images. The cross-correlation coefficient of these images with the original He I one shows a decrease with increasing noise. The computed noise and the corresponding decrease of the cross-correlation are presented in Table 4 and shown as error bars in Fig. 4. These results show that the decrease of the cross correlation is real and it is not due to noise.
Table 4. Fractional noise estimates and its influence on the cross-correlation.

\begin{tabular}{lcc}
\hline Ion & $\begin{array}{c}\text { Image } \\
\text { noise }(\%)\end{array}$ & $\begin{array}{c}\text { decrease in } \\
\text { cross-correlation }(\%)\end{array}$ \\
\hline He I & 5 & 1 \\
O IV & 8 & 3 \\
O V & 6 & 1 \\
Ne VI & 22 & 15 \\
Mg IX & 17 & 15 \\
Mg X & 21 & 15 \\
Si VIII & 38 & 38 \\
Fe XII & 34 & 38 \\
Fe XIV & 45 & 43 \\
Si XII & 31 & 27 \\
\hline
\end{tabular}

\subsection{Dopplershifts}

Figure 5 shows the intensity images of the $\mathrm{He}$ I and $\mathrm{O} v$ CDS lines, as well as the Si IV and Ne VIII SUMER lines, with the corresponding dopplergrams. It appears that many bright features correspond to redshifts, although there is not a one to one correspondence. We can clearly see that the dopplergrams present a different morphology from the intensity images, since they show much smaller structures.

In order to study the morphological characteristics in a more quantitative way, we computed the autocorrelation function for the intensity images and the dopplergrams. The autocorrelation function can give us information 

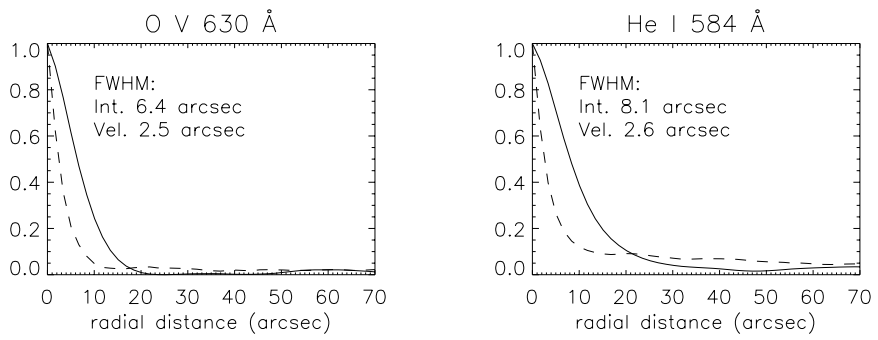

Si IV $1394 \AA$
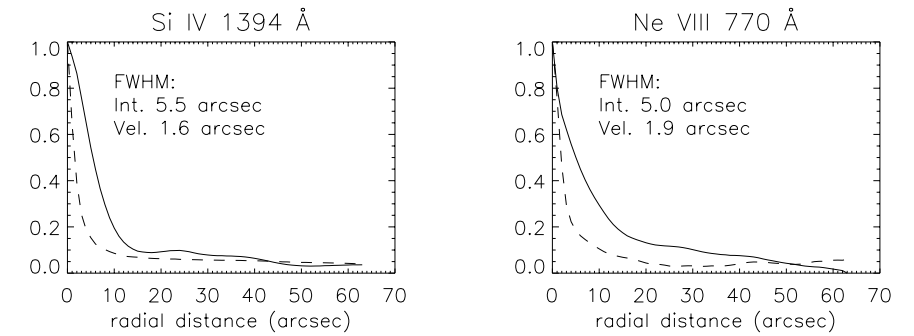

Fig. 6. Mean autocorrelation function for the intensity (continuous lines and the dopplergrams (dashed lines). The FWHM of the bright intensity structures of the network is larger than the one of the dopplergrams.

about the mean spatial scales of the structures of each image. We applied a cosine bell function at the image borders in order to smooth the discontinuity. After the two-dimensional autocorrelation function had been calculated, we computed its radial variation, integrating over the angle. The autocorrelation function was computed for the intensity images and the dopplergrams of the spectral lines of He I, O v, Si IV and Ne VIII. Figure 6 shows the average autocorrelation function for intensities and velocities for the four lines. We see that the intensity autocorrelation function is broader than the dopplergram one. This shows that the intensity structures are larger than those of the dopplergrams. On the average, the intensity $F W H M$ is twice that of the velocity curve. Patsourakos et al. (1999), following the same method of analysis, only for the intensities found values a little larger, but this may be due to the fact that the region we study is much closer to the limb. The differences we detected between the SUMER dopplergrams in different lines are comparable to the differences between CDS and SUMER dopplergrams. For both instruments the correlation between dopplergrams, deduced from lines of different temperature, is not high. This implies that there are velocity changes with height. One can only say that intense downflows or upflows in a dopplergram correspond to similar, even though not necessarily intense, velocities of a dopplergram from a line close in temperature.

In order to study the behaviour of the network and the internetwork regions separately, we calculated velocity histograms for each line, for the bright and dark features using the appropriate masks. Four of them, two for each instrument, are shown in Fig. 7. We can see that distributions are similar and there is no significant difference between those of CDS and of SUMER lines. The distributions of the dark features have a larger standard deviation.
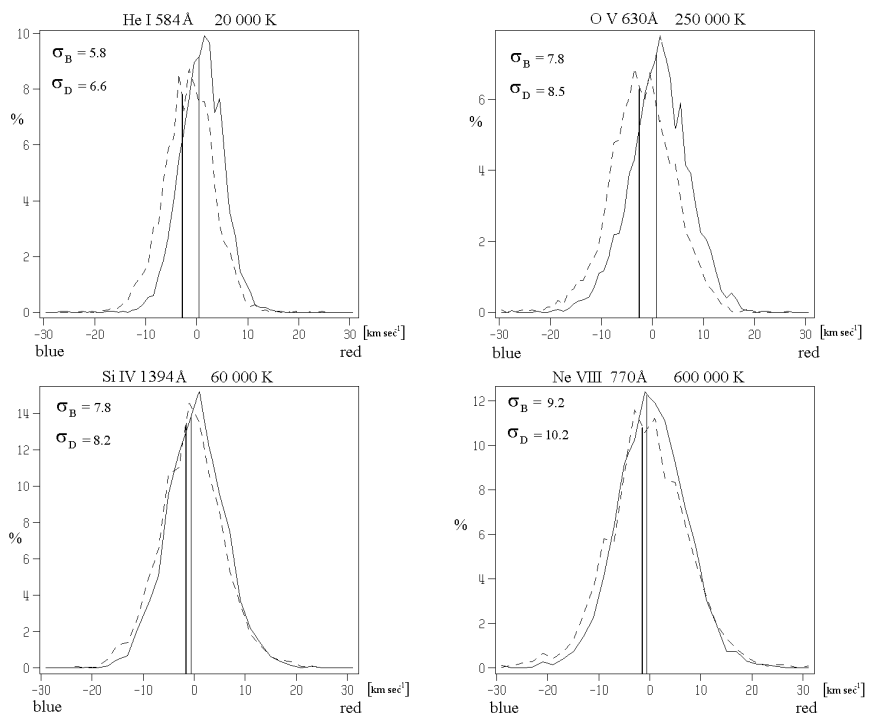

Fig. 7. Line of sight velocity distributions deduced from the $\mathrm{He} \mathrm{I}$ and $\mathrm{O} \mathrm{v}$ lines (CDS, first row), as well as from the Si IV and Ne VI lines (SUMER, second row). Continuous lines correspond to the bright features of the network and dashed lines to the internetwork dark features. Vertical lines mark the median values of the velocity (thick for the dark features and thin for the bright ones). At the upper left part of each graph we show the standard deviation $\left(\sigma_{\mathrm{B}}\right.$ for the bright and $\sigma_{\mathrm{D}}$ for the dark features) for each distribution in $\mathrm{km} \mathrm{s}^{-1}$.

Table 5. Standard deviation of histograms.

\begin{tabular}{lrcc}
\hline Ion & Temperature $[\mathrm{K}]$ & $\sigma_{\mathrm{B}}\left[\mathrm{km} \mathrm{s}^{-1}\right]$ & $\sigma_{\mathrm{D}}\left[\mathrm{km} \mathrm{s}^{-1}\right]$ \\
\hline \hline He I & 20000 & 5.8 & 6.6 \\
Si IV & 60000 & 7.8 & 8.1 \\
O III & 100000 & 9.5 & 16.3 \\
O IV & 160000 & 10.3 & 16.8 \\
O V & 250000 & 7.8 & 8.5 \\
Ne VI & 400000 & 10 & $\ldots$ \\
Ne VIII & 630000 & 9.2 & 10.2 \\
Mg IX & 1000000 & 8.6 & $\ldots$ \\
\hline
\end{tabular}

The bright features are clearly redshifted with respect to the dark ones in all the lines. This can be seen by the redshift of the median in Fig. 7 which is $2.5 \mathrm{~km} \mathrm{~s}^{-1}$ for He I, $3.3 \mathrm{~km} \mathrm{~s}^{-1}$ for $\mathrm{O} \mathrm{V}, 0.9 \mathrm{~km} \mathrm{~s}^{-1}$ for Si IV and $0.8 \mathrm{~km} \mathrm{~s}^{-1}$ for Ne VIII. The relative redshift of the peak of the distributions is larger for the CDS lines He I and $\mathrm{O} \mathrm{V}$.

In addition to the histograms, we computed a scatter plot of velocities and line intensities for the $\mathrm{O} v(\mathrm{CDS})$ and the Si IV (SUMER) lines, presented in Fig. 8. We conclude that, despite the statistical errors, there is a weak but systematic tendency for the bright features to be redshifted.

As we already mentioned, there are velocity changes with height, implied by the differences of the dopplergrams from lines of different temperature. In Table 5 we give the formation temperature of each line and the corresponding $\mathrm{e}^{-1}$ half-width for the distributions of the bright $\left(\sigma_{\mathrm{B}}\right)$ and dark $\left(\sigma_{\mathrm{D}}\right)$ features. This quantity was derived by applying a gaussian fit to each histogram. For some spectral 

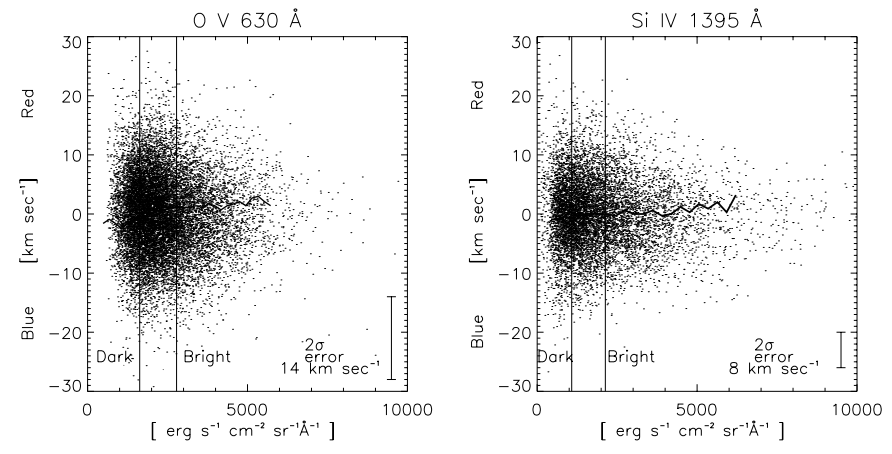

Fig. 8. Scatter plots between velocities and peak intensities of the $\mathrm{O} v$ line (CDS) and the Si IV line (SUMER). The solid line indicates the median. There is a small correlation, in both CDS and SUMER observations, between bright structures and redshift, implying that the brighter the structure the stronger the downflow.

lines, the half-width of the dark features is not indicated, because, due to the low counts of individual profiles, we cannot get reliable results. The half-widths of the bright features are smaller than those of the dark ones, a result which is in agreement with Athay \& Dere (1989), who found that the rms of the dopplershift distribution is enhanced for low intensity features in C IV $1548 \AA$ line. We notice an increase of the $\sigma$ with temperature up of about $10^{5.5} \mathrm{~K}$, but we cannot risk a generalization. This result is similar with the one found by Teriaca et al. (1999) for the non-thermal velocities. The $\mathrm{C}$ IV line at $100000 \mathrm{~K}$ is not included because, due to its blends ( $\mathrm{Si}$ I $1547.73 \AA$ and $1548.65 \AA$ ), the $\sigma$ computation is unreliable.

\section{Discussion}

Our observational findings can be summarized as follows.

The study of the morphology of the intensity images in different lines of the transition region, corresponding to different temperatures, showed a gradual disappearance of the network pattern and a decrease of the cross correlation with temperature. This should be real, since we proved that it is not significantly influenced by the noise.

In the low transition region the morphology in the intensity images is different from the morphology in the dopplergrams. It seems that the network intensity structures correspond to more than one structure in the dopplergrams, since they are smaller than the intensity ones. This can be clearly seen in Fig. 5, where intensity images and dopplergrams of the He I $584 \AA$, O v $630 \AA$ and Si IV $1394 \AA$ lines can be compared. Moreover, this is confirmed by the autocorrelation analysis (Fig. 6) which shows that the intensity-bright structures are roughly twice as large as the dopplershift features. This is in agreement with the results that Tsiropoula et al. (1994) found in $\mathrm{H} \alpha$ and Dere et al. (1984) in the C I $1561.4 \AA$ line. Dere et al. suggested that the small size of the flowing structures reflects the dominant role of the chromospheric jets, however they found similar structure size for intensity and veloc- ity features in C IV $1548 \AA$. This is in disagreement with our results, where the intensity structures are, in all the observed lines, larger than the ones of the corresponding dopplergrams. Brynildsen et al. (2001) present dopplergrams with larger scale features than the intensity ones, but they refer to active regions.

The study of the histograms of the velocities corresponding to the network and the internetwork regions of the images, shows that the network plasma is redshifted with respect to the internetwork one. The shifts of the histograms are very small, smaller than the error bar corresponding to each profile of the dopplergrams, but are systematic for all lines. This result is in agreement with those of other authors (e.g. Gebbie et al. 1981; Athay et al. 1983; Brynildsen et al. 1995, 1996, 1997, 1998a, 1998b) who find that high intensity profiles are more redshifted.

The only histogram which roughly shows absolute velocities is the one of the He I $584 \AA$ line, as it seems that the average dopplershift of the Sun at this wavelength is very near to zero $\left(0.2 \mathrm{~km} \mathrm{~s}^{-1} \pm 0.6 \mathrm{~km} \mathrm{~s}^{-1}\right.$ Peter \& Judge 1999, where positive values of the velocity mean a redshift). Peter (1999) mentions that the internetwork net blueshift observed in the He I line is due to the upward propagating shocks. This would imply that this line is formed below the magnetic canopy. Thus, for the He I line, the shift between the two histograms (Fig. 7) could be the result of upward motion in the internetwork and downward motion at the network boundaries. The corresponding dopplershift deduced from $\mathrm{O} v\left(9.8 \pm 1.0 \mathrm{~km} \mathrm{~s}^{-1}\right.$ Peter \& Judge 1999) and Si IV $\left(6.5 \pm 1.1 \mathrm{~km} \mathrm{~s}^{-1}\right.$ Peter \& Judge 1999) shows that the internetwork plasma in the transition region is also statistically redshifted but less than the network one. This would imply a different behaviour of the upper chromosphere and the low transition region. This result is also in agreement with the recent work of Peter (2000) for C IV, where he mentions that the redshifts are smaller in the internetwork than in the network region. Anyway, since the He I line is optically thick, and the emission processes are rather complicated and not fully understood yet (Andretta \& Jones 1997), this assumption should be verified with the study of other spectral lines (e.g. optically thin) formed at the same atmospheric level.

From Fig. 7 we can see the standard deviation of the network histogram is smaller than the standard deviation of the internetwork one. Since the standard deviation is an index of the velocity fluctuations, this may imply that there is a different mechanism for the network and the internetwork features. Moreover, we see that the standard deviation seems to be increasing with temperature, at least until about $160000 \mathrm{~K}$.

More observational work, with higher resolution, should be carried out to verify the bright-dark feature differences. In addition, the study of the multi-component nature of the line profiles (Peter 2000) will help in this direction. 
Acknowledgements. We would like to thank Dr. F. ChiuderiDrago and Dr. K. Bocchialini, for their contribution to the observation campaign, Dr. S. Patsourakos for his assistance with the data treatment, as well as Dr. V. Haugan and Dr. H. Peter for useful discussions. Last, but not least, we would like to thank our referee, Dr. P. G. Judge, for his crucial and important comments.

This research is supported in part by the Academy of Athens research program 200/480. The CDS project is supported by the United Kingdom PPARC. The SUMER project is financially supported by DLR, CNES, NASA and the ESA PRODEX program (Swiss contribution).

\section{References}

Achour, H., Brekke, P., Kjeldseth-Moe, O., \& Maltby, P. 1995, ApJ, 453, 945.

Alissandrakis, C. E., Drago, F., Bastian, T., et al. 1997, Advances in the physics of sunspots, ed. B. Schmieder, J. C. Toro del Iniesta, \& M. Vazquez, Proc. Astron. Soc. Pac. Conf. Ser.

Andretta, V., \& Jones, H. P. 1997, ApJ, 489, 375

Arnaud, M., \& Rothenflug, R. 1985, A\&AS, 60, 425

Arnaud, M., \& Raymond, J. 1992, ApJ, 398, 394

Athay, R. G., \& Dere, K. P. 1989, ApJ, 346, 514

Athay, R. G., Gurman, J. B., Henze, W., \& Shine, R. A. 1983, ApJ, 265, 519

Ayres, T. R., Stencel, R. E., Linsky, J. L., et al. 1983, ApJ, 274,801

Brekke, P. 1993, ApJ, 408, 735

Brekke, P., Hassler, D. M., \& Wilhelm, K. 1997, 175, 349

Bruner, E. C., Jr., Chipman, E. G., Lites, B. W., et al. 1976, ApJ, 210, L97

Brynildsen, N., Brekke, P., Fredvik, T., et al. 1998a, Solar Phys., 179, 279

Brynildsen, N., Brekke, P., Fredvik, T., et al. 1998b, Solar Phys., 181, 23

Brynildsen, N., Fredvik, T., Maltby, P., et al. 1997, Proceedings of the fifth SOHO workshop, ESA SP-404

Brynildsen, N., Kjeldseth-Moe, O., \& Maltby, P. 1995, ApJ, 455, L81

Brynildsen, N., Kjeldseth-Moe, O., \& Maltby, P. 1996, ApJ, 462,534

Chae, J., Yun, H. S., \& Poland, A. I. 1998, A\&AS, 114, 151

Cheng, C. C. 1991, in Mechanisms of Chromospheric and Coronal Heating, ed. P. Ulmschneider, E. R. Priest, \& R. Rosner (Berlin: Springer), 77

Chiuderi-Drago, F., Bocchialini, K., Lamartinie, S., et al. 1998, New perspectives on Solar Prominences, ed. D. F. Webb, B. Schmieder, \& D. M. Rust, Proc. Astron. Soc. Pac. Conf. Ser., 150, 55
Dammasch, I. E., Proceedings of the fourth ESO/ST-ECF Data Analysis Workshop, May 13-14, 1992, 137

Dammasch, I. E., Wilhelm, K., Curdt, W., \& Hassler, D. M. 1999, A\&A, 346, 294

Dere, K. P., Bartoe, J.-D. F., \& Brueckner, G. E. 1984, ApJ, 281,870

Domingo, V., Fleck, B., \& Poland, A. I. 1995, Solar Phys., 162,1

Doschek, G. A., Feldman, U., \& Bohlin, J. D. 1976, ApJ, 205, L117

Gebbie, K. B., Hill, F., Toomre, J., et al. 1981, ApJ, 251, L115

Gouttebroze, P., Vial, J.-C., Bocchialini, K., Lemaire, P., \& Leibacher, J. W. 1999, Solar Phys., 184, 253

Hassler, D. M., Dammasch, I. E., Lemaire, P., et al. 1999, Science, 283, 749

Hassler, D. M., Rottman, G. J., \& Orrall, F. Q. 1991, ApJ, 372,710

Harrison, R. A., et al. 1995, Solar Phys., 162, 233

Haugan, S. V. H. 1999, Solar Phys., 185, 275

Judge, P., Carlsson, M., \& Wilhelm, K. 1997, ApJ, 490, L195 Chromospheric and Rosner (Berlin: Springer), 615

Patsourakos, S., Vial, J.-C., Gabriel, A. H., \& Bellamine, N. 1999, ApJ, 522, 540

Peter, H., \& Judge, P. G. 1999, ApJ, 522, 1148

Peter, H. 1999, ApJ, 516, 490

Peter, H. 1999, ApJ, 522, L77

Peter, H. 2000, A\&A, 360, 761

Porter, J. G., Toomre, J., \& Gebbie, K. B. 1984, ApJ, 283, 879

Reeves, E. M. 1976, Solar Phys., 46, 53

Roussel-Dupré, D., \& Shine, R. A. 1982, Solar Phys., 77, 329

Rutten, R. J. 1999, in 3rd Advances in Solar Physics Euroconference: Magnetic Fields and Oscillations, ed. B. Schmieder, A. Hofmann, \& J. Staude, ASP Conf. Ser., 184, 181

Samain, D. 1991, A\&A, 244, 217

Spadaro, D., Antiochos, S. K., \& Mariska, J. T. 1991, ApJ, 382,338

Teriaca, L., Banerjee, D., \& Doyle, J. G. 1999, A\&A, 349, 636

Thompson, W. T., \& Brekke, P. 2000, Solar Phys., 195, 45

Thompson, W. T. 1998, CDS SOFTWARE NOTE No. 49, Deriving Statistics from NIS data.

Tsiropoula, G., Alissandrakis, C. E., \& Schmieder, B. 1994, A\&A, 290, 285

Wikstøl, Ø., Hansteen, V. H., Carlsson, M., \& Judge, P. G. 2000, ApJ, 531, 1150

Wilhelm, K., Curdt, W., Marsh, E., et al. 1995, Solar Phys., 162,189

Wood, B. E., Harper, G. M., Linsky, J. L., \& Dempsey, R. C. 1996 ApJ, 458, 761

Wood, B. E., Linsky, J. L., \& Ayres, T. R. 1997, ApJ, 478, 745 